\title{
Prosthodontic rehabilitation in an elderly patient with a hemi-maxillectomy defect
}

SADJ September 2019, Vol. 74 No. 8 p445 - p447

MM Mothopi-Peri', DG Howes ${ }^{2}$, CP Owen ${ }^{3}$

\section{CASE REPORT}

\section{Abstract}

Prosthodontic rehabilitation in patients with maxillectomy defects can be challenging. When rehabilitating with conventional means, problems of retention and stability are a common occurrence. Implant rehabilitation offers better outcomes in this regard but can be costly for the majority of patients especially in developing countries such as South Africa.

This treatment can also be contraindicated for patients with uncontrolled medical conditions such as diabetes mellitus. This case report illustrates a continuum of treatment in an elderly male patient with a hemi-maxillectomy defect. His successful management was made possible by positive changes that occurred in his life over a certain period of time.

\section{Keywords}

Maxillo-facial prosthodontics; maxillary defects; lifestyleappropriate treatment.

\section{INTRODUCTION}

Maxillectomy defects are caused by congenital diseases, cancers, trauma, burns and infections. Infections can be due to diseases such as diabetes mellitus and HIV/ AIDS. Rehabilitation of patients with maxillofacial defects poses challenges to both the clinician and the patient. Prosthodontic rehabilitation of elderly patients has been

\section{Author affiliations:}

1. Matshediso M Mothopi-Peri: BDS, MDent, Specialist, Department of Prosthodontics, School of Oral Health Science, Faculty of Health Sciences, University of the Witwatersrand.

ORCID Number: 0000-0001-8163-0642

2. Dale G Howes: $B S c, B D S, M D e n t, F C D(S A)$, Head, Department of Prosthodontics, School of Oral Health Science, Faculty of Health Sciences, University of the Witwatersrand. Currently at the University of Sydney, NSW, Australia.

ORCID Number: 0000-0001-6431-570

3. C Peter Owen: $B C h D, M S c D e n t, M C h D, F C D(S A)$, Professor Emeritus, Faculty of Health Sciences, University of the Witwatersrand. ORCID Number: 0000-0002-9565-8010

Corresponding author: $\mathrm{C}$ Peter Owen

Faculty of Health Sciences. 7 York Road, Parktown 2193, Johannesburg, South Africa.

Tel: +27 (0)83679 2205

Email: peter.owen@wits.ac.za

Author contributions:

1. Matshediso M Mothopi-Peri: Carried out the treatment and wrote the paper - $60 \%$

2. Dale G Howes: Supervised the treatment and revised the paper $-30 \%$

3. C Peter Owen: Consulted, co-wrote and revised the paper - $30 \%$ reported in the literature, and some authors have described patients with diseases such as diabetes mellitus. ${ }^{1}$

Treatment protocols ranged from conventional to implant supported prostheses. ${ }^{2}$ Most of these reports, however, failed to highlight the importance of other patient factors such as age, medical history, socio-economic status, biologic and psychological status.

The purpose of this case report is to present a continuum of treatment for an elderly diabetic patient with a hemimaxillectomy defect. It will illustrate the need for a dynamic treatment-planning approach in maxillofacial rehabilitation, mainly because of the possibility of changes in the patient's conditions and situations.

\section{CASE HISTORY}

A 71 year old male patient presented with a hemimaxillectomy defect as a result of a resection carried out as part of managing mucomycosis. During the surgical procedure he lost teeth from the last molar in the first quadrant to the canine in the second quadrant, also leaving him with a defect in the right maxillary buccal vestibule (Fig. 1) which caused an oroantral communication.

The patient's medical history included Type II diabetes mellitus, asthma, hypertension and urinary problems. At initial presentation he was on medication for all these conditions and had a frail appearance.

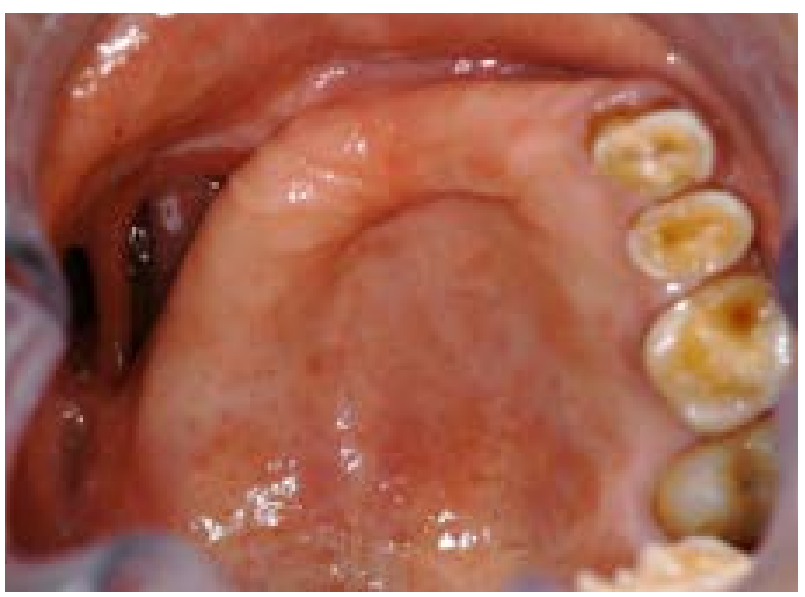

Figure 1. Maxillary arch after surgical resection. 
The defect and partial edentulousness caused difficulty in mastication, drinking, and speech; which in turn caused his withdrawal from social activities. Due to his medical condition and socio-economic status at the time, non-invasive 'appropriatech'3 treatment options were carried out for him.

A removable maxillary denture with acrylic obturation was constructed. Retention was not optimal, and over the next five years, three dentures/obturators had to be constructed (Fig. 2). This impacted negatively on the patient's daily activities and quality of life.

During this time the patient's medical condition improved and this warranted consideration for alternative treatment options which would improve his deteriorating oral condition. With motivation, funds were obtained which enabled implant planning and treatment.
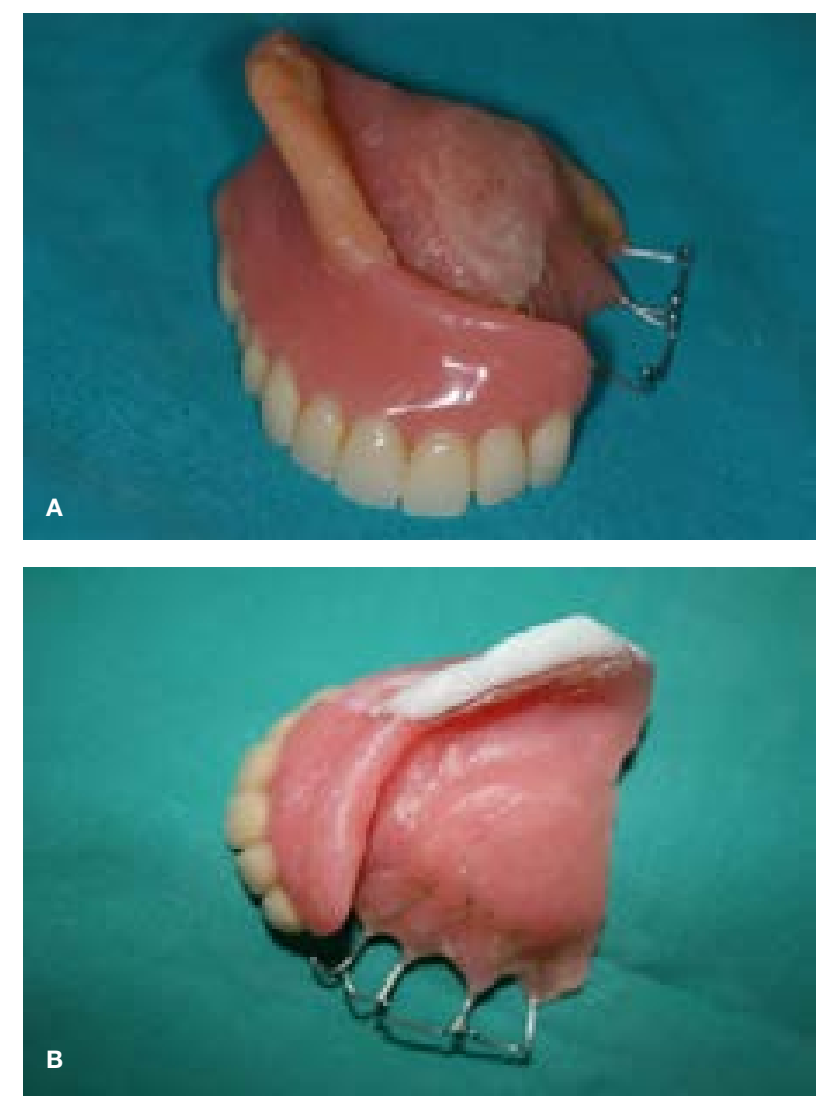

Figure 2. Examples of removable obturators.

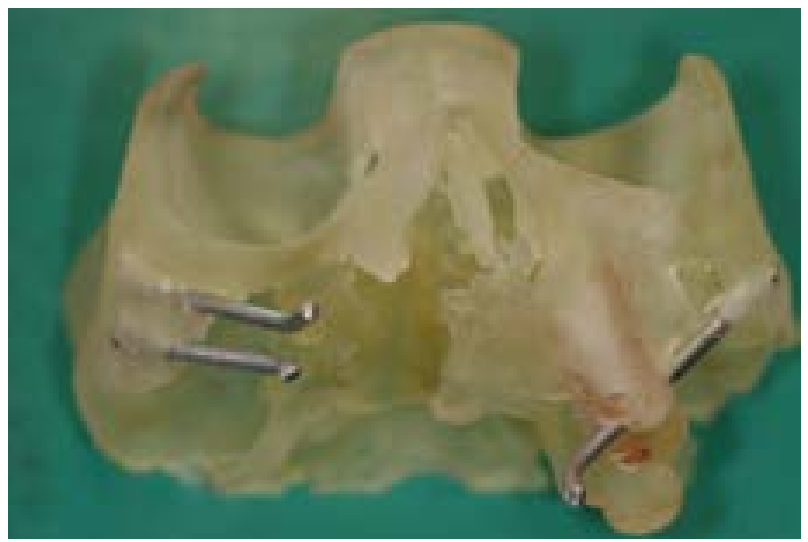

Figure 3. Rapid prototype model, showing positions of oncology implants on the right and the zygomatic implant on the left.
Planning was carried out using cone beam computed tomography, and the production of stereolithic models through rapid prototyping (Fig 3). It was decided to extract teeth in the second quadrant except for the third molar which was to be used as a guide to maintain the occlusal vertical dimension. Implants were placed as follows:

- Two oncology implants (Southern Implants, Irene, South Africa) in the right zygoma.

- One co-axis implant (Southern BAT 12d13) on the left side in the first premolar site.

- One zygomatic (Southern) implant on the left side. The patient was then rehabilitated with a bar retained overdenture (Fig. 4).
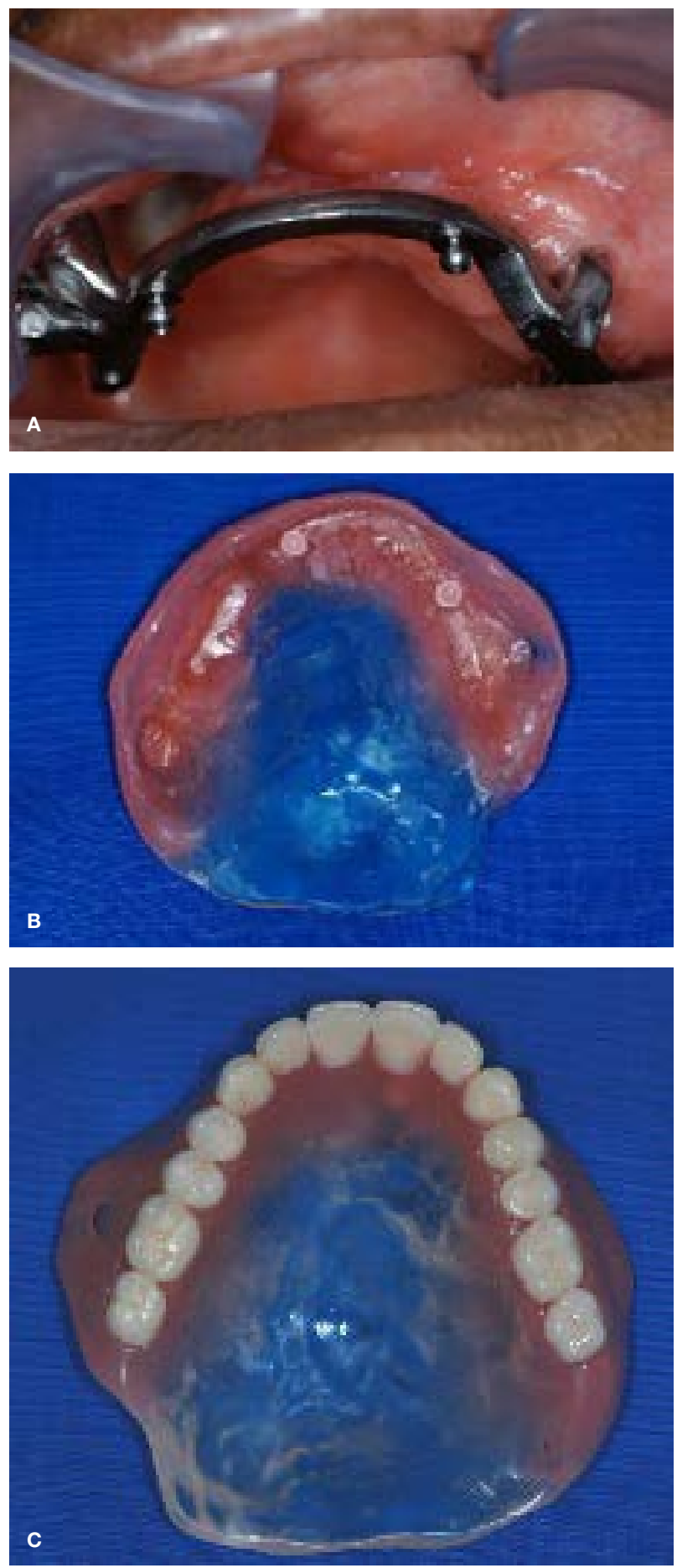

Figure 4. Bar-retained overdenture obturator. 
After a year the patient reported difficulty in manipulating the prosthesis and a lack of retention. The bar-retained prosthesis was then converted to a fixed implant supported prosthesis with a separate removable obturator (Fig. 5). This design allowed the obturator to be removed and cleaned without difficulty. ${ }^{4}$

\section{DISCUSSION}

Conventional means of rehabilitation are cost-effective and therefore affordable to the majority of patients, especially in a developing country. ${ }^{5}$ But they tend to have problems of stability and retention as exemplified in this patient, negatively affecting mastication, deglutition and speech.

Although implant rehabilitation is not a contraindication for elderly and diabetic patients, it is a prerequisite that patients are healthy and well controlled during treatment. ${ }^{1}$

The patient under discussion here benefited from the initial appropriatech cost-effective approach i.e. removable partial dentures (RPDs) with acrylic obturation. When he experienced problems with the RPDs and his health condition improved, implant rehabilitation was considered but could only happen when funds were made available. It was then possible to plan and place implants and rehabilitate with a bar-retained overdenture. When the patient experienced challenges with this, his prosthesis was converted to a fixed implant supported prosthesis with a separate removable acrylic palate/obturator.

\section{CONCLUSION}

This case is presented as an example of the dynamic nature of treatment planning, which allowed for a continuum of treatment to be carried out. It demonstrates the importance of versatility when treating patients in maxillofacial prosthodontics, not only between patients but within an individual as well ${ }^{6}$ so that treatment is patient mediated and appropriate to prevailing lifestyle and condition.

\section{References}

1. Balshi TJ. Dental implants in the diabetic patient: A retrospective study. Implant Dent. 1999; 8: 355-9.

2. Allen PF, McMillan AS. A longitudinal study of quality of life outcomes in older adults requesting implant prostheses and complete removable dentures. Clin Oral Implants Res. 2003; 14(2): 173-9.

3. Owen PC. Appropriatech: Prosthodontics for the many, not just for the few. Int J Prosthodont. 2004; 17: 261-2.

4. Boyes-Varley JG, Howes DG, Davidge-Pitts KD, Branemark I, McAlpine JA. A protocol for maxillary reconstruction following oncology resection using zygomatic implants. Int $\mathrm{J}$ Prosthodont. 2007; 20: 521-31.

5. Carlsson GE, Omar R. The future of complete dentures in oral rehabilitation. A critical review. Journal of Oral Rehabilitation 2010; 37: 143-56.

6. Mothopi MM, Owen CP, Howes DG, Naidoo LM. The need for versatility in the prosthodontic treatment of maxillofacial defects. SADJ 2012; 67(7): 420-3.
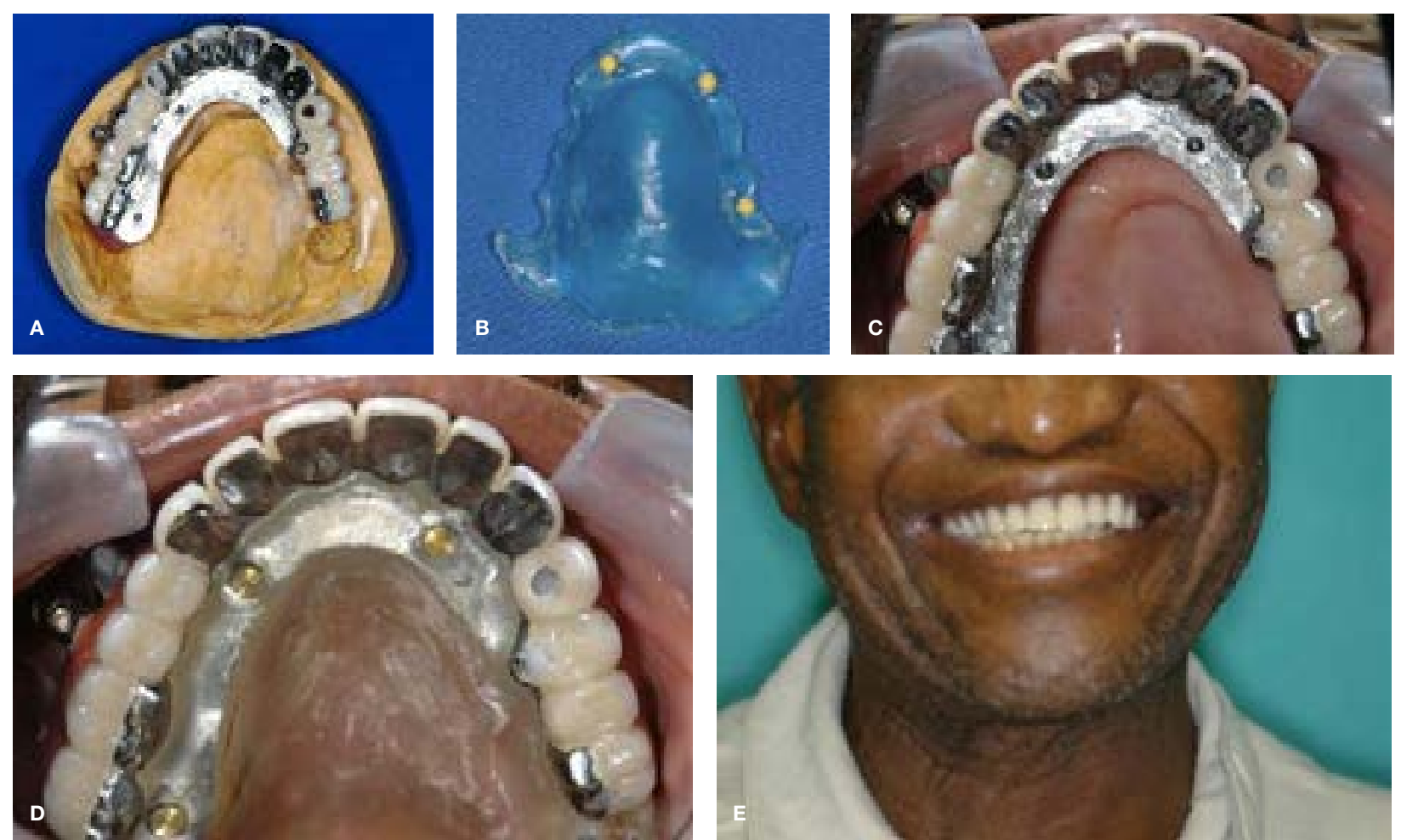

Figure 5. Fixed framework on model $(A)$; separate obturator $(B)$;

Framework in mouth $(C)$ and with obturator in place $(D)$; final smile $(E)$. 